\title{
3-Core Fiber Modal Analysis
}

\author{
David Benedicto, Juan Antonio Vallés \\ Group of Laser \& Optical Tecnologies (TOL) \\ Instituto de Investigación en Ingeniería de Aragón (I3A) \\ Universidad de Zaragoza, Mariano Esquillor s/n, 50018, Zaragoza, Spain. \\ Tel.+34-976762707, e-mail: 629816@unizar.es
}

\begin{abstract}
Multicore fibers (MCFs) are expected as a good candidate for overcoming the capacity limit of current optical communication systems. This paper describes the modal analysis of a 3-core MCF taking into account some geometrical and material degrees of freedom. A reasonable tailoring of the modal distribution is shown to be achievable.
\end{abstract}

\section{Introduction}

During the last twenty years, the transmission capacity per fiber in optical communication technologies has been increased by three orders of magnitude. Recently, single mode single core fiber (SM-SCF) transmission systems have achieved a transmission capacity up to $100 \mathrm{~Tb} / \mathrm{s}$ and a capacitydistance product over $100 \mathrm{~Pb} / \mathrm{s} \cdot \mathrm{km}$ as shown in Fig. 1.

However, there seems to be a fundamental limit around $100 \mathrm{~Tb} / \mathrm{s}$, and therefore the capacity of existing standard SM-SCF may no longer satisfy the growing capacity demand. Among the practical systems maximum capacity limiting factors are the "fiber fuse" phenomenon, which causes permanent thermo-chemical damage to fiber cores for long distance propagation, nonlinear interactions due to various optical nonlinear effects, and the optical bandwidth for optical amplification.

In order to further increase the capacity of an optical fiber, space division multiplexing (SDM) has been proposed. The spatial degree of freedom can be exploited by transmitting over a set of different SMF, over multi-core fibers (MCF), or over different modes of a few-mode fiber (FMF). Mode division multiplexing (MDM) is also expected to break this capacity barrier, by using the multiplicity of modes which can propagate in specially designed FMF. MCF is expected to be a good candidate since both core and mode multiplicities can be combined.

\section{Results and discussion}

In this paper, we focus on the modal analysis of a 3core MCF by varying three parameters of the fiber: the difference between the cladding and the core indices, the distance between cores and the the core radius.

In the following calculation, the MCF is assumed to be based on a step-index design with a core diameter of $5 \mu \mathrm{m}$, cladding index of 1.55 , core index of 1.56, and the three cores are equidistant and centered in a circumference of $10 \mu \mathrm{m}$ diameter.

Starting with the assumed values, we have computed the modal field distribution and the effective index of the propagating modes. The $x$ component electric field distribution of the fundamental mode is shown in Fig. 2, together with that of the second propagating mode, which is degenerated.

By raising the index difference or by lowering the distance between cores, it is possible to increase the difference between the effective indices of the fundamental mode and other propagating modes. This behavior is useful when a quasi-single mode fiber is required. The effect of increasing the distanceor lowering the index difference causes the opposite response, making each of the three modes to propagate with a similar effective index, as it is shown in Fig. 3.

Up to now, we have assumed that each core corresponds to a single mode fiber. In a single mode fiber, increasing the size of the core causes the fiber to allow more propagation modes. In the same sense, by increasing the size of each core in the MCF we allow another propagating mode, as it is shown in Fig. 4. Fig. 5 shows the evolution of the effective index for the fundamental and fourth propagation modes as a function of the core radius. Once reached a $6.5 \mu \mathrm{m}$ core radius, the fourth mode effective index becomes higer than the background index and the mode starts to propagate. 


\section{Conclusions}

By means of this modal analysis, it is posible to select the index difference between the core and the cladding, the core radius and the separation between cores of a 3-core MCF in order to accomplish particular requirements in terms of the effective index of the propagation modes.

\section{REFERENCES}

[1]. TOSHIO MORIOKA. New generation optical infrastructure technologies: "EXAT initiative" towards 2020 and beyond. Presented at the OptoElectronics and Communications Conf., Hong Kong, China, 2009, paper FT4.

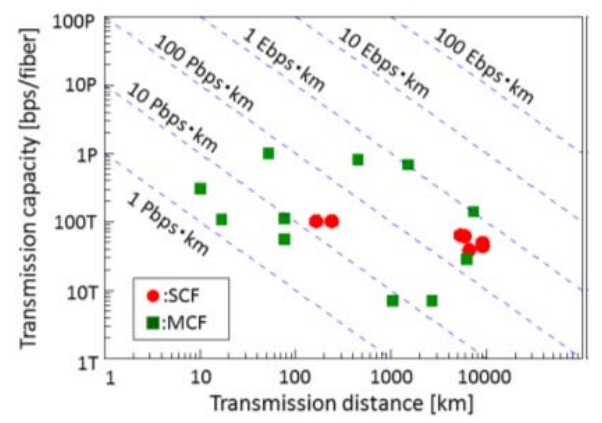

Figure 1: Recently reported transmission capacity and transmission distance by using single-mode single-core fibers and single-mode multicore fibers.
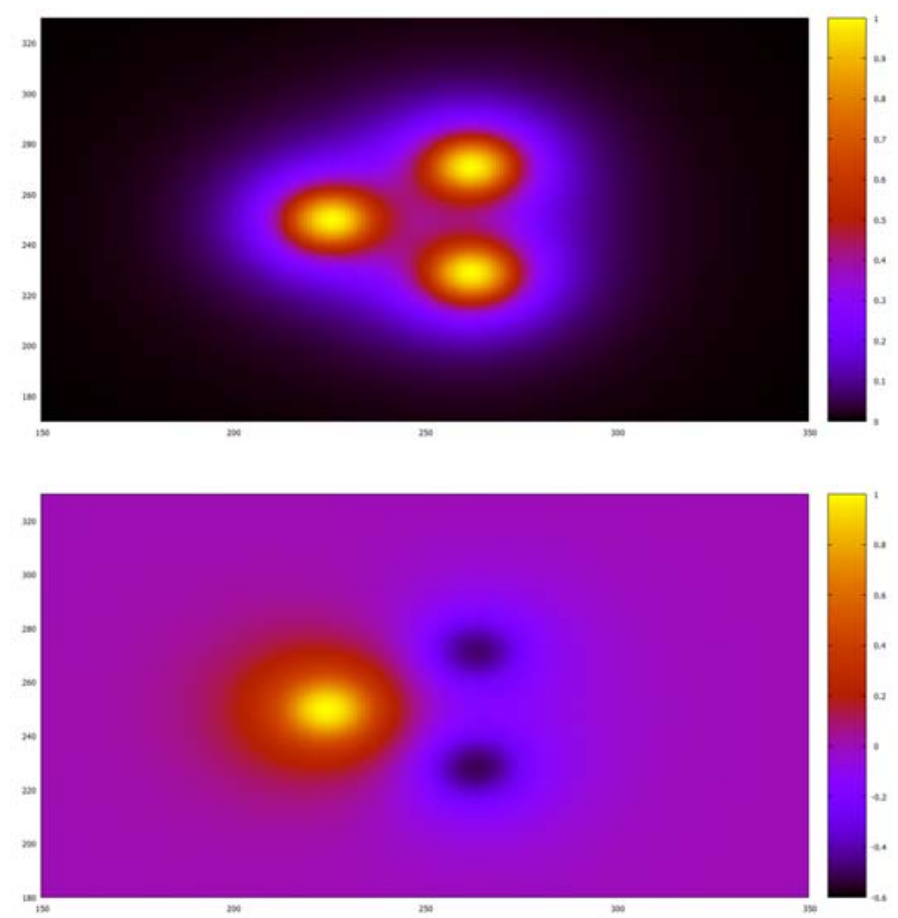

Figure 2: Normalized mode field distributions of the $x$-component of the electric field for the fundamental and second propagation modes.
[2]. KUNIMASA S. and SHOICHIRO M. Multicore Fiber Technology. Journal of light technology, Vol. 34, No. 1, January 1, 2016., pp 54-66.

[3]. TAKAYUKI M., HIDEHIKO T., AKIHIDE S. and YUTAKA M. Dense space division multiplexed transmission systems using multi-core and multimode fiber. Journal of lightwave technology, Vol 34, No 2, January 14, 2015, pp 582-592.

[4]. ROLAND R., NICOLAS K., and RENE-JEAN E. Spot-based mode couplers for mode-multiplexed transmission in few-mode fiber, IEE Photonics technology letters, Vol. 34, No. 21, November 1, 2012, pp 1973-1976.

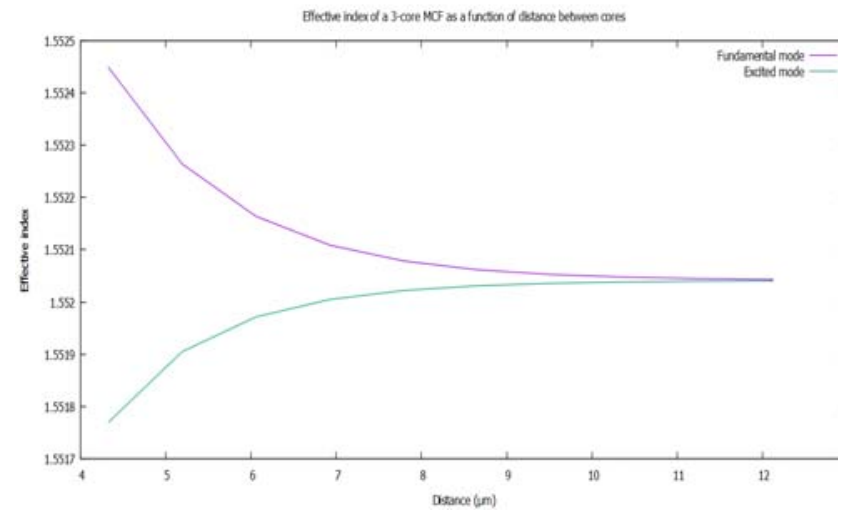

Figure 3: Effective index as a function of the distance between cores for the fundamental and second propagation modes.

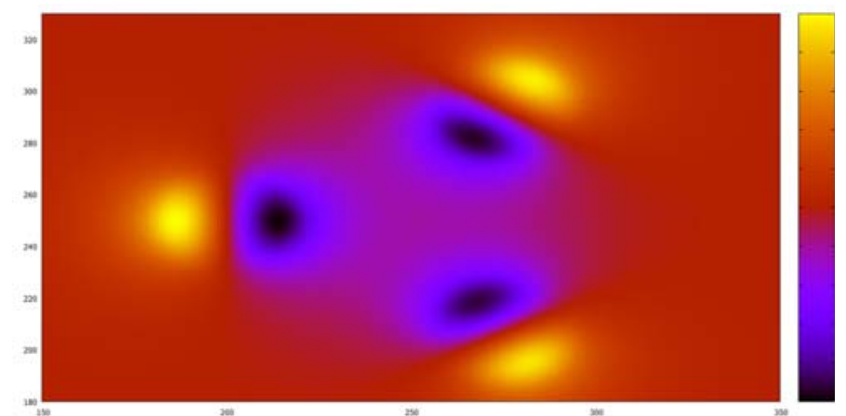

Figure 4: Normalized mode field distributions of the $x$ component of the electric field for the fourth propagation mode.

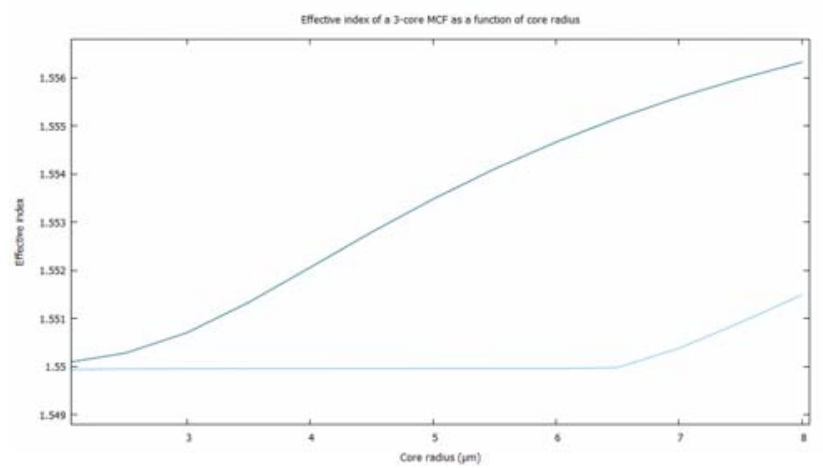

Figure 5: Effective index as a function of the radius of the core for the fundamental and fourth propagation modes. 